\title{
Excess floppy modes and multibranched mechanisms in metamaterials with symmetries
}

\author{
Luuk A. Lubbers 1 and Martin van Hecke \\ Huygens-Kamerlingh Onnes Laboratory, Universiteit Leiden, P.O. Box 9504, NL-2300 RA Leiden, The Netherlands \\ and AMOLF, Science Park 104, 1098 XG Amsterdam, The Netherlands
}

(Received 19 October 2018; published 21 August 2019)

\begin{abstract}
Floppy modes - deformations that cost zero energy - are central to the mechanics of a wide class of systems. For disordered systems, such as random networks and particle packings, it is well-understood how the number of floppy modes is controlled by the topology of the connections. Here we uncover that symmetric geometries, present in, e.g., mechanical metamaterials, can feature an unlimited number of excess floppy modes that are absent in generic geometries, and in addition can support floppy modes that are multibranched. We study the number $\Delta$ of excess floppy modes by comparing generic and symmetric geometries with identical topologies, and show that $\Delta$ is extensive, peaks at intermediate connection densities, and exhibits mean-field scaling. We then develop an approximate yet accurate cluster counting algorithm that captures these findings. Finally, we leverage our insights to design metamaterials with multiple folding mechanisms.
\end{abstract}

DOI: 10.1103/PhysRevE.100.021001

Floppy modes (FMs) play a fundamental role in the mechanics of a wide variety of disordered physical systems, from elastic networks [1-7] to jammed particle packings [8-10]. Floppy modes also play a role in many engineering problems, ranging from robotics to deployable structures, where the goal is to design structures that feature one or more mechanisms [11]. Mechanisms are collections of rigid elements linked by flexible hinges, designed to allow for a collective, floppy motion of the elements. More recently, floppy modes and mechanisms have received renewed attention in the context of mechanical metamaterials, which are architected materials designed to exhibit anomalous mechanical properties, including negative response parameters, shape morphing, and self-folding [6,7,12-22]. An important design strategy for mechanical metamaterials borrows the geometric design of mechanisms, and replaces their hinges by flexible parts which connect stiffer elements [22]. In all these examples, understanding how the geometric design controls the number and character of the floppy modes plays a central role.

For systems consisting of objects with a total of $n_{d}$ degrees of freedom, connected by hinges that provide $n_{c}$ constraints, the number of nontrivial floppy modes $n_{f}$ and states of self-stress $n_{s s}$ are related by Maxwell-Calladine counting as $n_{f}-n_{s s}=n_{d}-n_{c}-n_{r b}$, where $n_{r b}$ counts the trivial rigid body modes ( $n_{r b}=3$ in two dimensions) [23]. For generic, disordered systems $n_{f}$ and $n_{s s}$ can be determined separately from the connection topology [1,2], but when symmetries are present such approaches break down and counting only yields the difference $n_{f}-n_{s s}$. For example, spring lattices which feature perfectly aligned bonds can generate excess floppy modes (and associated states of self-stress) that disappear under generic perturbations and thus escape topologybased counting methods [1,2,24-29]. Mechanical metamaterials often feature symmetric architectures where excess floppy modes (EFMs) may arise, but their geometries are more complex than spring lattices [22,30-35].
We focus on understanding the EFMs of a geometry which underlies a range of metamaterials [14,16,17,20-22,36,37]: rigid quadrilaterals connected by flexible hinges. We define $n_{s}$ and $n_{g}$ as the number of nontrivial floppy modes for symmetric systems consisting of squares, and stress-free generic systems obtained by randomly displacing the corners of linked squares with magnitude $\epsilon=0.1$ [38]. Each quadrilateral has three degrees of freedom (DOF), and in a fully connected lattice each quadrilateral has four connections in the bulk, and less near the boundary. By counting the degrees of freedom and constraints one finds that $M \times N$ lattices of generic quadrilaterals are rigid $\left(n_{g}=0\right)$ when $M \geqslant 3$ and $N \geqslant 3$; however, replacing the generic quadrilaterals by equally sized squares, such lattices always exhibit an EFM where the squares can counter-rotate [14], i.e., $n_{s}=1$ [Fig. 1(a)]. Here we address two key issues. First, what is the multiplicity and statistics of EFMs in diluted lattices as considered recently [21,36,37] [Fig. 1(b)]? Second, do EFMs in diluted lattices possess anomalous properties, and if so, how can we leverage these to embed new functionalities into metamaterials?

System and methods. We consider diluted $N \times N$ lattices of quadrilaterals connected by springs of unit stiffness and zero (a)

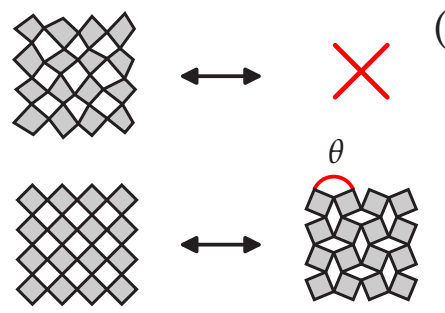

(b)

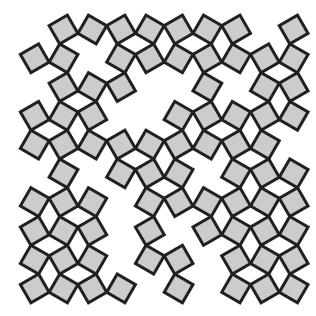

FIG. 1. (a) $N \times N$ systems of generic quadrilaterals are rigid for $N \geqslant 3$, but have a floppy, "hinging" mode characterized by the opening angle $\theta \in[0, \pi]$ for perfectly symmetric squares. (b) Diluted square tiling $(N=10, \rho=0.8)$. 


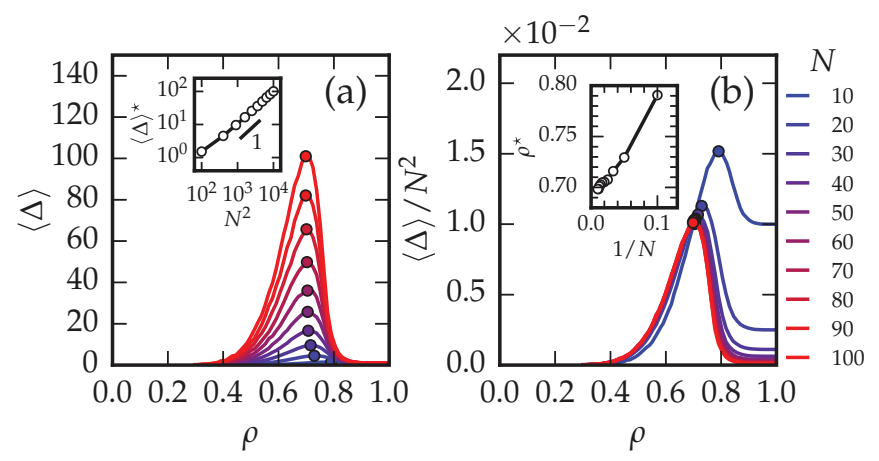

FIG. 2. (a) Average number of excess floppy modes as a function of the filling fraction $\rho$ for $N$ as indicated. Inset: the peak value $\langle\Delta\rangle^{\star} \approx \beta N^{2}$ with $\beta \approx 0.01$. (b) Scaling collapse of $\langle\Delta\rangle / N^{2}$ for large $N$. Inset: peak position $\rho^{\star}$ as a function of $1 / N$ shows clear convergence of $\rho^{\star}$ to $0.69 \pm 0.01$ for large $N$.

rest length - unless noted otherwise, we use open boundary conditions [Fig. 1(b)]. For each filling fraction $\rho$, we repeatedly remove $(1-\rho) N^{2}$ random quadrilaterals (or links; see below) to obtain a specific connection topology, and for each topology we calculate $n_{s}$ and $n_{g}$.

Random dilution. We focus on the ensemble averaged number of EFMs, $\langle\Delta\rangle:=\left\langle n_{s}-n_{g}\right\rangle$ as a function of $\rho$ [Fig. 2(a)] [39]. In the dilute limit (small $\rho$ ), the system breaks up into isolated quadrilaterals for which $n_{s}=n_{g}$ and $\langle\Delta\rangle=0$. In the undiluted limit $(\rho=1)$ discussed above, there are zero $\left(n_{g}=0\right)$ floppy modes in the generic case and a single $\left(n_{s}=1\right)$ counter-rotating EFM in the symmetric case, so that $\langle\Delta\rangle=1$. Strikingly, we find that for intermediate densities, $\langle\Delta\rangle$ is not monotonic but exhibits a maximum $\langle\Delta\rangle^{*} \gg 1$, unambiguously evidencing the emergence of multiple EFMs in diluted, symmetric systems. To see how $\langle\Delta\rangle$ can become larger than 1, consider $m$ disconnected, fully filled $3 \times 3$ clusters: as each symmetric cluster has an internal hinging EFM absent for generic clusters, $\Delta=m$ for such a hypothetical system. While more intricate topologies arise for random dilution, we will show that the internal hinging motion of clusters of squares are central to EFMs.

Scaling. We find that the number of EFMs, $\langle\Delta\rangle$, follows mean-field scaling. First, we find that its maximum, $\langle\Delta\rangle^{*}$, grows linearly with $N^{2}$, which implies that the peak density of EFMs, $\beta:=\langle\Delta\rangle^{*} / N^{2}$, is a constant [inset Fig. 2(a)]. Second, we can collapse our data as $\langle\Delta\rangle / N^{2}=f(\rho)$, with the peak location approaching a constant as $\rho^{\star}=\rho_{0}+\alpha / N$ with $\rho_{0} \approx 0.69, \alpha \approx 1.00$ [Fig. 2(b)]. Third, we found that the distribution of $\Delta$ at fixed $\rho$ is Gaussian (not shown). Finally, we have also studied random bond removal, i.e., removal of individual links, and find a very similar scaling collapse and peak location. All this strongly suggests that EFMs occur with a constant density and finite correlation length [40].

Clusters and counting. The number of floppy modes in generic systems, $n_{g}$, can exactly be determined by the pebble game [1,2], and therefore to study $\Delta$ one can study $n_{s}$ and vice versa. We now construct a counting argument for the number of floppy modes in the symmetric systems $\left(n_{s}\right)$. In this argument we interpret diluted geometries as systems of $N_{c}$ clusters and connectors. We define clusters as groups (a)

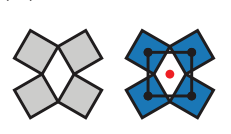

(c)

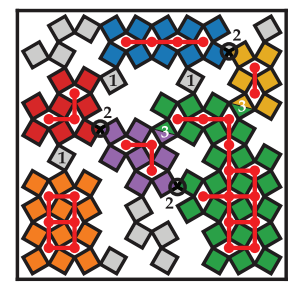

(b)

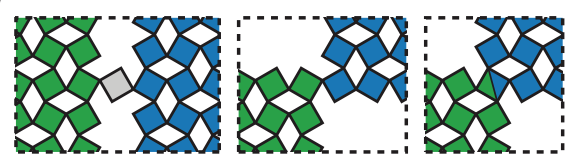

(d)

(e)
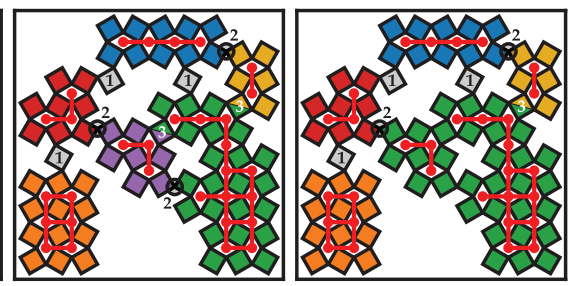

FIG. 3. (a) A 4-block is formed by four connected quadrilaterals, and can be identified by filling a (red) dot on the dual grid. (b) Type-1, type-2, and type-3 connectors. (c) Clusters are adjacent 4-blocks, and feature straight connections between dots on the dual grid. All quadrilaterals that belong to a single cluster share a color; quadrilaterals belong to zero (gray), one (color), or two clusters (bicolor). Gray quadrilaterals that are connected via two hinges at clusters are type- 1 connectors; all other gray quadrilaterals are "remaining quadrilaterals", which are either isolated, connected to other gray quadrilaterals only, or connected at only one side to a cluster. (d) Clusters in a pruned system where all remaining quadrilaterals are removed. (e) Cluster merging; note that merged clusters may contain 4-blocks that are not adjacent.

of adjacent 4-blocks, where 4-blocks are groups of four quadrilaterals connected in a loop [Fig. 3(a)]. Each cluster has a single internal hinging mode in addition to their three rotational and translational floppy modes. Clusters can be connected by three types of connectors [see Fig. 3(b)]: a type- 1 connection introduces one constraint and consists of a single quadrilateral; a type- 2 connection introduces two constraints and occurs when two adjacent clusters touch; a type-3 connection introduces three constraints and occurs when two clusters share a quadrilateral.

Generally, a number of loosely connected remaining quadrilaterals are neither connector nor part of any cluster [Fig. 3(c)]. We remove these quadrilaterals and focus on pruned systems that solely consist of clusters and connectors [Fig. 3(d)], and refer to the quantities in the pruned systems by accents (e.g., $\Delta^{\prime}=n_{s}^{\prime}-n_{g}^{\prime}$ )-later we will present evidence that $\Delta^{\prime}$ and $\Delta$ are very close.

In the simplest counting arguments one ignores the states of self-stress, leading to an estimate $\Delta_{0}=n_{s}^{\prime}-n_{g}^{\prime}$. We now consider that each cluster has three global and one internal degree of freedom, and estimate $n_{s}^{\prime}$ via the difference of the number of degrees of freedom associated with the clusters, $4 N_{c}$, and the number of constraints between clusters, $\Sigma_{i j} C_{i j} / 2$, where the connection number $C_{i j}:=n_{1, i j}+2 n_{2, i j}+3 n_{3, i j}$ counts the number of constraints between clusters $i$ and $j$, and $n_{k, i j}$ denotes the number of type- $k$ connectors. Noting that we need to subtract the three rigid body motions, this yields $n_{s}^{\prime}=4 N_{c}-\Sigma C_{i j} / 2-3$, and $\Delta_{0}:=4 N_{c}-\Sigma C_{i j} / 2-3-n_{g}^{\prime}$. However, we will show that $\Delta_{0}$ is quite different from $\Delta^{\prime}$ obtained numerically, as symmetries lead to degeneracies between connectors. 
(a)
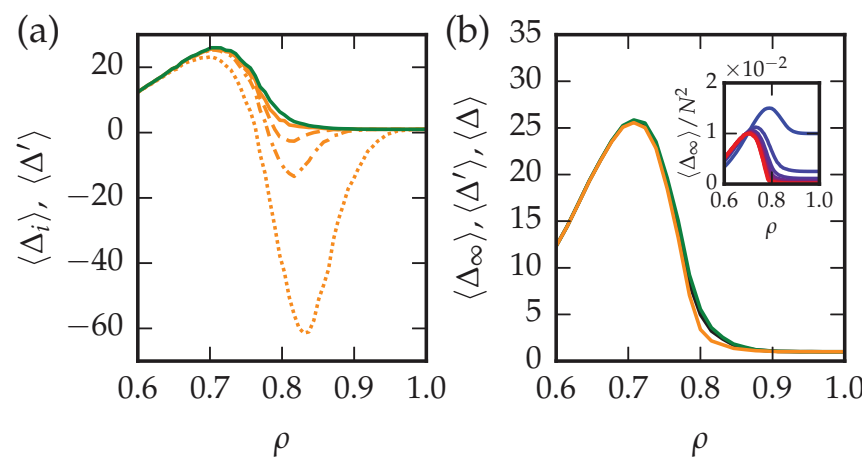

FIG. 4. (a) Counting predictions $\left\langle\Delta_{0}\right\rangle,\left\langle\Delta_{1}\right\rangle,\left\langle\Delta_{2}\right\rangle$ (orange dotted, dash-dotted, dashed) and $\left\langle\Delta_{\infty}\right\rangle$ (solid orange) compared to the exact result $\left\langle\Delta^{\prime}\right\rangle$ (green) for $N=50$. (b) Comparison of $\left\langle\Delta_{\infty}\right\rangle$ (orange), $\left\langle\Delta^{\prime}\right\rangle$ (green), and $\langle\Delta\rangle$ (black) for $N=50$. Inset: asymptotic scaling collapse of $\left\langle\Delta_{\infty}\right\rangle$ for $N=10,20, \ldots, 90$ (blue to red).

Degenerate constraints arise when $C_{i j}>4$. To see how such degeneracies arise, consider a system of two clusters. Without connections these have a total of eight DOFs, but even if $C_{i j}$ is very large, this system must feature at least three rotational and translational modes and one global hinging mode, so that for $C_{i j}>4$, constraints must be redundant [40]. To take these degeneracies into account, we define a sequence of increasingly accurate predictions $\Delta_{0}, \Delta_{1}, \ldots, \Delta_{\infty}$ by progressively eliminating degeneracies. To define $\Delta_{1}$, we merge all pairs of clusters for which $C_{i j} \geqslant 4$, and then for the resulting set of clusters and connectivities define $N_{c}^{1}, C_{i j}^{1}$, and define $\Delta_{1}:=4 N_{c}^{1}-\Sigma C_{i j}^{1} / 2-3-n_{g}^{\prime}$. As cluster merging may yield new pairs of heavily connected clusters, we iterate this procedure to obtain $\Delta_{2}, \Delta_{3}, \ldots$ Eventually, no cluster merging is possible when all $C_{i j}^{n}<4$ [Fig. 3(e)], and the final result for $\Delta_{n}$ is denoted as $\Delta_{\infty}$.

Results. We now compare the results $\left\langle\Delta_{n}\right\rangle$ obtained by our iterative cluster counting argument to the numerical results for $\left\langle\Delta^{\prime}\right\rangle$ and $\langle\Delta\rangle$ (Fig. 4). We find that while $\left\langle\Delta_{0}\right\rangle$ captures the peak in the number of EFMs, it fails in the high density regimes where degeneracies are abundant. Cluster merging significantly improves the results, and $\left\langle\Delta_{\infty}\right\rangle$ is found to be within a few percent of $\left\langle\Delta^{\prime}\right\rangle$ [Fig. 4(a)]. Moreover, we find that pruning has a minor effect, as $\left\langle\Delta_{\infty}\right\rangle,\left\langle\Delta^{\prime}\right\rangle$, and $\langle\Delta\rangle$ are very close [Fig. $4(\mathrm{~b})]$. Finally, $\left\langle\Delta_{\infty}\right\rangle$ displays a scaling collapse closely matching that of $\langle\Delta\rangle$ [inset Fig. 4(b)]. We conclude that our approach of cluster merging and counting is able to accurately capture the numerically observed data for the number of EFMs, thus providing fresh insight into how floppy modes and states of self-stress proliferate in complex geometries with symmetries.

Multibranch planar folding mechanisms. Shape-morphing metamaterials can act as deployable structures, deforming along a well-defined path under external actuation [11,1922,30,41-44]. Their geometric design often follows from a mechanism with a single floppy mode. To embed multiple shape changes in a metamaterial, rather than designing structures with multiple floppy modes which lead to a continuous family of shapes, mode competition, and frustration $[21,45,46]$, a better route may be to consider structures with a single floppy mode but multiple, discrete branches.
Multibranch mechanisms occur in, e.g., origami [30,35], but we are not aware of any two-dimensional mechanisms that allow multibranch behavior without self-intersections. Here we show that by leveraging the symmetries in systems of hinging squares one can design multibranched mechanisms, consisting of connected clusters whose magnitude of hinging is coupled, while the signs of the hinging motion of the clusters can only take on a limited number of values.

To understand the design of multibranched mechanisms, we first consider the hinging motion of a single cluster. We define the opening angle $\psi:=(\theta-\pi / 2) / 2$, color the squares according to their alternating rotating motion, and note that we can distinguish four link types, associated with connections at the north, east, south, or west tip of a dark square [Fig. 5(a)]. The crucial observation is that the distance between two links is invariant under opening angle inversion $(\psi \leftrightarrow-\psi)$ if and only if both links are of equal type [Fig. 5(a)]. Specifically, if we consider links that are vertically aligned, such as $A, B$, and $C$ in Fig. 5(a), we find that $A B$ is symmetric in $\psi$, while $A C$ is not [Fig. 5(b)].

We obtain multibranched mechanisms by connecting clusters by type- 2 connectors located at hinges $A$ and $B$, whose distance is symmetric in the opening angle. We define $\psi_{1}, \psi_{2}, \ldots$ such that for a global hinging mode all opening angles are equal. The symmetry of $A B(\psi)$ then implies that, e.g., the clusters 1 and 2 can be in a "homogeneous" state where $\psi_{1}=\psi_{2}$, and in a second state, where $\psi_{1}=$ $-\psi_{2}$ [47]. Clusters that can be connected by two type-2 connectors can easily be constructed, and strips of $m$ of such coupled clusters form a compound structure with one continuous hinging degree of freedom, and precisely $2^{m-1}$ discrete branches, where $\left\{\psi_{i}\right\}=\left\{\psi_{1},(-1)^{s_{2}} \psi_{1} \ldots,(-1)^{s_{m}} \psi_{1}\right\}$; here the binary variables $\left\{s_{2}, \ldots, s_{m}\right\}$ characterize the different branches [Fig. 5(c)].

More complex situations where the number of branches is different from a power of two arise when clusters are connected in more complex topologies. We designed a cluster which features the single and double-bump edge structures along both its vertical and horizontal edges, and connect these into a $2 \times 2$ lattice [Fig. 5(d)]. As the (horizontal) connections between clusters 1 and 2, respectively 3 and 4, are of a different link type than the (vertical) connections between clusters 1 and 3 , respectively 2 and 4 , the sign of $\psi_{i}$ has to be consistent along either rows or columns. Fixing $\psi_{1}>0$, we obtain two consistent row configurations with $\left\{s_{i}\right\}=\{0,0,0,0\}$ and $\{0,0,1,1\}$; similarly, consistent column configurations are $\left\{s_{i}\right\}=\{0,0,0,0\}$ and $\{0,1,0,1\}$. It is easy to show that these are all allowed possibilities, and as we double count the homogeneous configuration, this example constitutes a three-branch mechanism. We note that the table of allowed branches $s_{2}, s_{3}$ is equivalent to the truth table of a NAND gate. Extending this design to $m \times n$ tilings, we obtain $2^{m-1}$ different column arrangements, $2^{n-1}$ different row arrangements yielding $2^{m-1}+2^{n-1}-1$ branches. For small $m \leqslant 3$ and $n \leqslant 3$ this already allows one to make mechanisms with $1,2,3,4,5,7,8,9,11$, and 15 branches. This suggests that our design approach, where multiple blocks with symmetric motions are connected together, and where looplike compatibility conditions determine which branches 

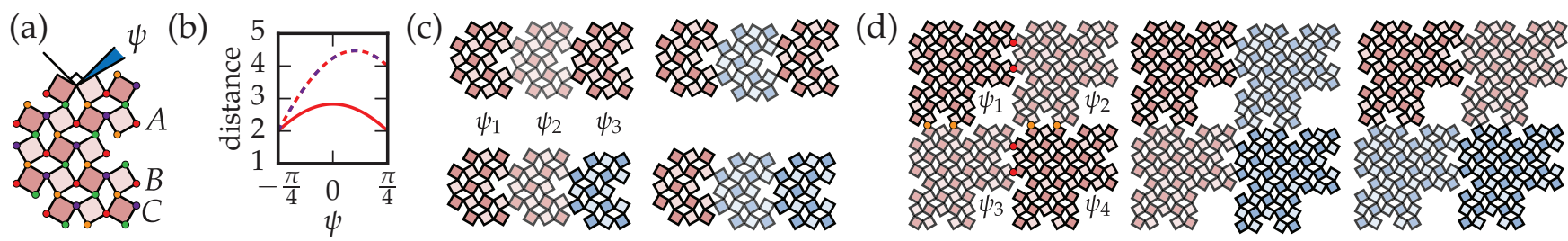

FIG. 5. (a) A cluster with positive opening angle $\psi:=(\theta-\pi / 2) / 2$. The colored dots indicate the four types of hinges. (b) The distance $A B$ (full) is symmetric in $\psi$ as $A$ and $B$ correspond to the same hinge type $[A B=2 \sqrt{2} \cos (\psi)]$ while $A C$ (dashed) is not invariant under $\psi \leftrightarrow-\psi$, as $A$ and $C$ correspond to different hinge types $[A C=A B+2 \sin (\psi+\pi / 4)]$. (c) Clusters connected by type- 2 connectors at points of the same hinge type can either have equal or opposite $\psi$ (red: $\psi>0$; blue: $\psi<0$ ). Fixing $\psi_{1}>0$ to break the global inversion symmetry $\psi_{i} \leftrightarrow-\psi$, the four branches of motion are characterized by the freely assignable signs of $\psi_{2}$ and $\psi_{3}$. (d) Compound cluster structure consisting of four identical unit cells that exhibits three branches of motion (see text and [40]).

can be realized, constitutes a powerful method to design complex, multibranched mechanisms.

Outlook and discussion. We have characterized the excess floppy modes that arise in diluted lattices of square elements, and have introduced an approximate yet accurate methodology to identify and count such excess modes. As our method is developed and tested for square elements, it would be interesting to see if it is similarly successful for other systems with symmetries, including diluted triangular and kagome lattices. Furthermore, we used our insights to design planar mechanisms with multiple discrete folding motions. Such mechanisms, which feature a single continuous degree of freedom but multiple discrete branches, provide new design avenues for (soft) robotics, deployable structures, and mechanical metamaterials [11,22,48,49], as well as open up systematic strategies for the design of multistable structures [30]. Finally, open questions include how one designs compounds with any integer number of branches, or with arbitrary "truth" tables for the hinging signs of the clusters $\left\{s_{i}\right\}$.

Acknowledgments. We acknowledge discussion with W. Ellenbroek, B. G.-g. Chen, and S. Guest, and funding from the Netherlands Organization for Scientific Research through a VICI Grant No. NWO-680-47-609.
[1] D. J. Jacobs and M. F. Thorpe, Phys. Rev. Lett. 75, 4051 (1995).

[2] D. J. Jacobs and M. F. Thorpe, Phys. Rev. E 53, 3682 (1996).

[3] S. Alexander, Phys. Rep. 296, 65 (1998).

[4] W. G. Ellenbroek, Z. Zeravcic, W. van Saarloos, and M. van Hecke, Europhys. Lett. 87, 34004 (2009).

[5] W. G. Ellenbroek, V. F. Hagh, A. Kumar, M. F. Thorpe, and M. van Hecke, Phys. Rev. Lett. 114, 135501 (2015).

[6] J. W. Rocks, N. Pashine, I. Bischofberger, C. P. Goodrich, A. J. Liu, and S. R. Nagel, Proc. Natl. Acad. Sci. USA 114, 2520 (2017).

[7] D. R. Reid, N. Pashine, J. M. Wozniak, H. M. Jaeger, A. J. Liu, S. R. Nagel, and J. J. de Pablo, Proc. Natl. Acad. Sci. USA 115, E1384 (2018).

[8] C. S. O’Hern, L. E. Silbert, A. J. Liu, and S. R. Nagel, Phys. Rev. E 68, 011306 (2003).

[9] M. van Hecke, J. Phys.: Condens. Matter 22, 033101 (2010).

[10] A. J. Liu and S. R. Nagel, Annu. Rev. Condens. Matter Phys. 1, 347 (2010).

[11] E. T. Filipov, T. Tachi, and G. H. Paulino, Proc. Natl. Acad. Sci. USA 112, 12321 (2015).

[12] R. S. Lakes, Science 235, 1038 (1987).

[13] G. W. Milton, J. Mech. Phys. Solids 40, 1105 (1992).

[14] J. N. Grima and K. E. Evans, J. Mater. Sci. Lett. 19, 1563 (2000).

[15] R. S. Lakes, T. Lee, A. Bersie, and Y. C. Wang, Nature (London) 410, 565 (2001).

[16] T. Mullin, S. Deschanel, K. Bertoldi, and M. C. Boyce, Phys. Rev. Lett. 99, 084301 (2007).
[17] K. Bertoldi, M. C. Boyce, S. Deschanel, S. M. Prange, and T. Mullin, J. Mech. Phys. Solids 56, 2642 (2008).

[18] J. Shim, C. Perdigou, E. R. Chen, K. Bertoldi, and P. M. Reis, Proc. Natl. Acad. Sci. USA 109, 5978 (2012).

[19] C. Coulais, E. Teomy, K. de Reus, Y. Shokef, and M. van Hecke, Nature (London) 535, 529 (2016).

[20] C. Coulais, C. Kettenis, and M. van Hecke, Nat. Phys. 14, 40 (2018).

[21] C. Coulais, A. Sabbadini, F. Vink, and M. van Hecke, Nature (London) 561, 512 (2018).

[22] K. Bertoldi, V. Vitelli, J. Christensen, and M. van Hecke, Nat. Rev. Mater. 2, 17066 (2017).

[23] C. Calladine, Int. J. Solids Struct. 14, 161 (1978).

[24] E. Guyon, S. Roux, A. Hansen, D. Bideau, J. P. Troadec, and H. Crapo, Rep. Prog. Phys. 53, 373 (1990).

[25] S. D. Guest and J. Hutchinson, J. Mech. Phys. Solids 51, 383 (2003).

[26] S. D. Guest and P. W. Fowler, Philos. Trans. R. Soc. A 372, 20120029 (2014).

[27] W. G. Ellenbroek and X. Mao, Europhys. Lett. 96, 54002 (2011).

[28] T. C. Lubensky, C. L. Kane, X. Mao, A. Souslov, and K. Sun, Rep. Prog. Phys. 78, 073901 (2015).

[29] X. Mao and T. C. Lubensky, Annu. Rev. Condens. Matter Phys. 9, 413 (2018).

[30] S. Waitukaitis, R. Menaut, Bryan Gin-ge Chen, and M. van Hecke, Phys. Rev. Lett. 114, 055503 (2015).

[31] K. Bertoldi, P. M. Reis, S. Willshaw, and T. Mullin, Adv. Mater. 22, 361 (2010). 
[32] J. T. B. Overvelde, S. Shan, and K. Bertoldi, Adv. Mater. 24, 2337 (2012).

[33] J. Shim, S. Shan, A. Košmrlj, S. H. Kang, E. R. Chen, J. C. Weaver, and K. Bertoldi, Soft Matter 9, 8198 (2013).

[34] K. Bertoldi, Annu. Rev. Mater. Res. 47, 51 (2017).

[35] M. B. Pinson, M. Stern, A. C. Ferrero, T. A. Witten, E. Chen, and A. Murugan, Nat. Commun. 8, 15477 (2017).

[36] Y. Tang, G. Lin, L. Han, S. Qiu, S. Yang, and J. Yin, Adv. Mater. 27, 7181 (2015).

[37] D. Rayneau-Kirkhope, C. Zhang, L. Theran, and M. A. Dias, Proc. Phys. Soc. London, Sec. A 474 (2018).

[38] This perturbation is sufficiently large so that in generic systems the eigenvalues of EFMs become large and numerically clearly separated from those of FMs. See Supplemental Material [40] for details about the floppy mode identification.

[39] We note that $n_{s}$ and $n_{g}$ are almost two orders of magnitude larger than $\Delta$, and that moreover, $n_{s}$ and $n_{g}$ have their peaks at significantly lower densities than $\Delta$. See Supplemental Material [40] for a demonstrating graph.
[40] See Supplemental Material at http://link.aps.org/supplemental/ 10.1103/PhysRevE.100.021001 for details.

[41] G. W. Milton and A. V. Cherkaev, J. Eng. Mater. Technol. 117, 483 (1995).

[42] Z. You and S. Pellegrino, Int. J. Solids Struct. 34, 1825 (1997).

[43] M. Schenk and S. D. Guest, Proc. Natl. Acad. Sci. USA 110, 3276 (2013).

[44] L. H. Dudte, E. Vouga, T. Tachi, and L. Mahadevan, Nat. Mater. 15, 583 (2016).

[45] S. Shan, S. H. Kang, P. Wang, C. Qu, S. Shian, E. R. Chen, and K. Bertoldi, Adv. Funct. Mater. 24, 4935 (2014).

[46] J. T. B. Overvelde, J. C. Weaver, C. Hoberman, and K. Bertoldi, Nature (London) 541, 347 (2017).

[47] Configurations $\left\{\psi_{i}\right\}$ and $\left\{-\psi_{i}\right\}$ are related by a global sign inversion symmetry and are considered to be on the same branch.

[48] D. Rus and M. T. Tolley, Nature (London) 521, 467 (2015).

[49] J. T. B. Overvelde, T. A. De Jong, Y. Shevchenko, S. A. Becerra, G. M. Whitesides, J. C. Weaver, C. Hoberman, and K. Bertoldi, Nat. Commun. 7, 10929 (2016). 